\title{
Melatonin regulates L-arginine transport and NADPH oxidase in young rats with bile duct ligation: role of protein kinase $C$
}

\author{
You-Lin Tain'2, Chih-Cheng Chen', Chien-Te Lee', Ying-Hsien Kao', Jiunn-Ming Sheen', Hong-Ren Yu' and Li-Tung Huang'
}

\begin{abstract}
BACKGROUND: Bile duct ligation (BDL) is a commonly used cholestatic liver disease (CLD) model. We recently found that L-arginine levels were significantly raised by melatonin in young rats with $B D L$. We hypothesized that protein kinase $C-\alpha$ $(P K C-\alpha)$ is involved in the increases of L-arginine in melatonintreated $\mathrm{BDL}$ rats. In addition, we tested whether melatonin prevents nicotinamide adenine dinucleotide phosphate (NADPH) oxidase-induced reactive oxygen species (ROS) production, in rats with $B D L$, through PKC.
\end{abstract}

METHODS: Four groups of young male rats were studied: shams $(n=6)$, untreated $\mathrm{BDL}$ rats $(n=9)$, melatonin-treated shams $(n=6, M)$, and melatonin-treated BDL rats $(n=6, \mathrm{BDL}+$ M). Melatonin-treated rats received daily melatonin $1 \mathrm{mg} / \mathrm{kg} / \mathrm{d}$ via i.p. injection. All surviving rats were killed $14 \mathrm{~d}$ after surgery.

RESULTS: Melatonin prevented BDL-induced mortality and kidney injury. Melatonin additionally increased L-arginine concentrations in $\mathrm{BDL}$ liver, which is correlated with decreased PKC- $\alpha$ translocation. Next, melatonin increased L-arginine levels in BDL kidneys, which was correlated with decreased renal levels of arginase II. In the BDL kidney, melatonin decreased PKC- $\beta$ translocation, reduced p47phox translocation, and diminished NADPH-dependent superoxide production.

CONCLUSION: Melatonin inhibits PKC $-\alpha$ to increase cationic amino acid transporter-1 (CAT-1)-mediated L-arginine uptake in $\mathrm{BDL}$ liver, whereas it inhibits PKC- $\beta$ to reduce NADPHdependent superoxide production.

M ost children with progressive cholestatic liver disease (CLD) can be cured only by liver transplantation. Bile duct ligation (BDL) is a commonly used CLD model. We have previously shown that melatonin lessens BDL-induced mortality and systemic oxidative damage in young rats through suppressing increased asymmetric dimethylarginine (ADMA) and oxidative stress $(1,2)$. Melatonin, an endogenously produced indoleamine secreted by the pineal gland, has the ability to scavenge reactive oxygen species (ROS) and reactive nitrogen species (3). The direct free radical scavenging effect of melatonin is independent of receptors; however, melatonin-mediated protective effect has been attributed to its receptor-dependent effect on the nitric oxide (NO) pathway (4).
L-Arginine, a substrate for $\mathrm{NO}$ synthase to generate NO, is mainly derived from protein catabolism $(85 \%)$ or from $\mathrm{L}^{-}$ citrulline by argininosuccinate synthase (ASS) and argininosuccinate lyase (ASL) (5). The transport of L-arginine is mainly mediated by the cationic amino acid transporter (CAT) family (5). We demonstrated that modulation of L-arginine metabolism by melatonin might have protective effects on rats with $\mathrm{BDL}$ (2). In BDL rats, we found that melatonin might restore ROS/NO balance to prevent BDL-induced kidney injury and death (2). Intriguingly, in rats with BDL, L-arginine levels were significantly raised by melatonin in both the liver and kidney. However, the detailed mechanisms whereby melatonin modulates $\mathrm{L}$-arginine transport and metabolism remain unclear.

Protein kinase $\mathrm{C}(\mathrm{PKC})$ is involved in melatonin-mediated signaling pathways $(6,7)$. PKC signaling is also involved in the activation of nicotinamide adenine dinucleotide phosphate (NADPH) oxidase and oxidative liver damage $(8,9)$. PKC is a superfamily of serine-threonine kinases and incorporates 12 isoforms, including conventional PKC isoforms $(\alpha$, $\beta I, \beta I I, \gamma)$. Several contributions have investigated the role of conventional PKC in the regulation of $\mathrm{L}$-arginine metabolism, although with contradicting results (10-13). Yet most of these studies suggest that activation of PKC- $\alpha$ inhibits CAT- 1 for the delivery of L-arginine. In addition, PKC- $\alpha$ can enhance superoxide production via NADPH oxidase assembly with the p47phox subunit (8). Similarly, another conventional PKC isoform, PKC- $\beta$, can increase p47phox translocation and induce fibrosis $(14,15)$.

Melatonin selectively regulates the different PKC isoforms $(16,17)$; only PKC- $\alpha$ and $-\beta$ are expressed in the liver and kidneys (18); both PKC- $\alpha$ and PKC- $\beta$ have been reported to regulate NADPH oxidase assembly $(8,14)$; and the CAT- 1 isoform is mainly responsible for L-arginine transport. Therefore, we assume that PKC- $\alpha$ and PKC- $\beta$ are differentially regulated by melatonin on CAT-mediated $\mathrm{L}$-arginine transport and NADPH oxidase-induced ROS production in the liver and kidney in rats with BDL.

Most children with CLD can be cured only by undergoing liver transplantation. In this regard, a better understanding of the potential therapy to retard CLD progression is of supreme

'Department of Pediatrics, Kaohsiung Chang Gung Memorial Hospital and Chang Gung University College of Medicine, Kaohsiung, Taiwan; ${ }^{2}$ Center for Translational Research in Biomedical Sciences, Kaohsiung Chang Gung Memorial Hospital and Chang Gung University College of Medicine, Kaohsiung, Taiwan; ${ }^{3}$ Department of Nephrology, Kaohsiung Chang Gung Memorial Hospital and Chang Gung University College of Medicine, Kaohsiung, Taiwan; ${ }^{4}$ Department of Medical Research, E-DA Hospital, I-Shou University, Kaohsiung, Taiwan. Correspondence: Li-Tung Huang (huang_li@pie.com.tw) 
Table 1. Morphological and biochemical values in different experimental groups

\begin{tabular}{|c|c|c|c|c|}
\hline & Sham & BDL & $M$ & $\mathrm{BDL}+\mathrm{M}$ \\
\hline Mortality & $0 \%(0 / 6)$ & $33.3 \%(3 / 9)$ & $0 \%(0 / 6)$ & $0 \%(0 / 6)$ \\
\hline Body weight (g) & $94 \pm 5$ & $88 \pm 3^{*}$ & $110 \pm 4^{*+}$ & $80 \pm 3^{*}$ \\
\hline $\begin{array}{l}\text { Left kidney } \\
\text { weight } / 100 \mathrm{~g} \text { BW }\end{array}$ & $0.70 \pm 0.02$ & $0.79 \pm 0.07^{*}$ & $0.67 \pm 0.02^{\dagger}$ & $0.84 \pm 0.02^{*}$ \\
\hline $\begin{array}{l}\text { Liver weight/ } \\
100 \mathrm{~g} \mathrm{BW}\end{array}$ & $4.94 \pm 0.33$ & $7.07 \pm 0.78^{*}$ & $4.76 \pm 0.15^{\dagger}$ & $6.87 \pm 0.10^{*}$ \\
\hline $\begin{array}{l}\text { Total bilirubin } \\
\text { (mg\%) }\end{array}$ & $0.38 \pm 0.05$ & $4.87 \pm 0.61^{*}$ & $0.35 \pm 0.04^{+}$ & $4.72 \pm 0.17^{*}$ \\
\hline $\begin{array}{l}\text { Direct bilirubin } \\
(\mathrm{mg} \%)\end{array}$ & $0.12 \pm 0.01$ & $4.25 \pm 0.54^{*}$ & $0.11 \pm 0.01^{\dagger}$ & $3.68 \pm 0.16$ \\
\hline AST (U/I) & $101 \pm 6$ & $401 \pm 54^{*}$ & $116 \pm 13^{+}$ & $368 \pm 30^{*}$ \\
\hline $\mathrm{ALT}(\mathrm{U} / \mathrm{I})$ & $56 \pm 3$ & $91 \pm 10^{*}$ & $51 \pm 4^{+}$ & $108 \pm 9^{*}$ \\
\hline $\begin{array}{l}\text { Creatinine } \\
(\mathrm{mg} / \mathrm{dl})\end{array}$ & $0.31 \pm 0.05$ & $0.52 \pm 0.04^{*}$ & $0.29 \pm 0.06^{+}$ & $0.35 \pm 0.03^{\dagger}$ \\
\hline
\end{tabular}

$A L T$, alanine transaminase; $A S T$, aspartate transaminase; $B D L$, bile duct ligation; $B D L+M$, melatonin-treated $\mathrm{BDL}$ rat; $\mathrm{BW}$, body weight; $\mathrm{M}$, melatonin-treated sham.

${ }^{*} P<0.05$ vs. sham. ${ }^{\dagger} P<0.05$ vs. BDL.

clinical importance. This study was therefore designed to address two specific questions: first, to examine whether PKC- $\alpha$ is involved in the increases of $\mathrm{L}$-arginine in the liver and kidneys in melatonin-treated BDL rats and second, to test whether melatonin prevents NADPH oxidase-induced ROS production through $\mathrm{PKC}$ in rats with $\mathrm{BDL}$.

\section{RESULTS}

\section{General Morphological Features and Biochemistry}

As shown in Table 1, the mortality rate for the BDL group was $33 \%$ (3/9). Both BDL and BDL + melatonin-treated sham (M) groups had similar lesser body weight, whereas the $\mathrm{M}$ group displayed greater body weight as compared with the sham group. Both the BDL and the BDL + M groups showed renal and hepatic hypertrophy (expressed as an increased tissue weight to body weight ratio). Consistent with our previous study findings $(1,2)$, BDL resulted in extrahepatic cholestasis, which was confirmed by increases in plasma aspartate transaminase, alanine transaminase, and direct and total bilirubin levels. Plasma creatinine level was higher in the BDL group than in the sham group, whereas melatonin treatment prevented the increase.

\section{L-Arginine and ADMA Levels in the Plasma, Liver, and Kidneys}

As shown in Table 2, plasma L-arginine levels were not different among the four groups. Although the plasma L-arginine levels in BDL rats tended to be decreased, they did not reach significance. Plasma ADMA levels were elevated in the BDL group relative to the sham group; this was prevented by melatonin. Because L-arginine and ADMA compete for NO synthase, ARG:ADMA ratio has been used to represent NO bioavailability. Plasma ARG:ADMA ratios were similarly elevated in the $\mathrm{M}$ and $\mathrm{BDL}+\mathrm{M}$ groups as compared with the BDL group. In the liver, $\mathrm{L}$-arginine levels were higher in the $\mathrm{BDL}$ and $\mathrm{BDL}+$ $M$ groups as compared with the sham group. ADMA concentrations in the liver were lower in $\mathrm{M}$ group than those in $\mathrm{BDL}$
Table 2. Plasma and tissue L-arginine and ADMA levels

\begin{tabular}{|c|c|c|c|c|}
\hline & Sham & $\mathrm{BDL}$ & M & $\mathrm{BDL}+\mathrm{M}$ \\
\hline \multicolumn{5}{|l|}{ Plasma ( $\mu \mathrm{mol})$} \\
\hline L-Arginine & $63.9 \pm 10.5$ & $46.4 \pm 3.8$ & $59.7 \pm 16.7$ & $60.2 \pm 10.9$ \\
\hline ADMA & $0.55 \pm 0.08$ & $0.65 \pm 0.1^{*}$ & $0.4 \pm 0.01^{* \dagger}$ & $0.45 \pm 0.03^{\dagger}$ \\
\hline ARG:ADMA ratio & $99.1 \pm 7.2$ & $85.3 \pm 10.3$ & $163.8 \pm 35.9^{\dagger}$ & $123.4 \pm 19.5^{\dagger}$ \\
\hline \multicolumn{5}{|c|}{ Liver ( $\mu \mathrm{mol} / \mathrm{mg}$ protein) } \\
\hline L-Arginine & $68.2 \pm 4$ & $89.4 \pm 8.9^{*}$ & $72.9 \pm 4.6$ & $99.9 \pm 4.9^{*}$ \\
\hline ADMA & $19.5 \pm 2.6$ & $24.7 \pm 3.4$ & $8.2 \pm 0.4^{*+}$ & $16 \pm 2.5^{\S}$ \\
\hline ARG:ADMA ratio & $3.76 \pm 0.49$ & $2.88 \pm 0.25$ & $9.06 \pm 0.75^{* \dagger}$ & $7.03 \pm 1.13^{* \dagger}$ \\
\hline \multicolumn{5}{|c|}{ Kidney ( $\mu \mathrm{mol} / \mathrm{mg}$ protein) } \\
\hline L-Arginine & $207 \pm 15.6$ & $271.5 \pm 21.1$ & $182.3 \pm 32$ & $342.6 \pm 23.1^{*+\S}$ \\
\hline ADMA & $21.5 \pm 2.3$ & $34.1 \pm 7.9$ & $15.7 \pm 3.4$ & $18.6 \pm 2.7$ \\
\hline ARG:ADMA ratio & $9.79 \pm 0.45$ & $9.27 \pm 1.46$ & $11.93 \pm 0.41$ & $19.71 \pm 2.63^{* \dagger}$ \\
\hline
\end{tabular}

group. Hepatic ARG:ADMA ratios were higher in the $\mathrm{M}$ and $\mathrm{BDL}+\mathrm{M}$ groups as compared with BDL group as well as the sham group. Similar to the liver, both renal $\mathrm{L}$-arginine levels and ARG:ADMA ratios were increased in the $\mathrm{BDL}+\mathrm{M}$ group as compared with the sham and BDL groups.

\section{L-Arginine Pathway in the Liver and Kidneys}

We further investigated whether melatonin increases L-arginine availability because of increased uptake by CAT- 1 and whether PKC- $\alpha$ and PKC- $\beta$ isoforms are involved. We found that the hepatic CAT-1 protein level (Figure 1a) was increased in the BDL rats (Figure $1 b$ ). Membranous PKC- $\alpha$ and PKC- $\beta$ protein levels in the liver were lower in BDL rats than those in sham rats (Figure 1a, c,d). Melatonin therapy significantly increased the PKC- $\alpha$ and PKC- $\beta$ protein levels in the cytosolic fraction but not the membranous fraction, indicating that melatonin did not increase their translocation. Moreover, the membranous PKC- $\alpha$ levels were significantly decreased in the BDL + $\mathrm{M}$ group as compared with those in BDL group. In the kidney, protein levels of CAT- 1 and PKC- $\alpha$ (Figure 2a) were not different among the four groups (Figure $2 \mathbf{b}, \mathbf{c}$ ). The renal cytosolic PKC- $\beta$ protein level was higher in the BDL group than in the sham group. Similarly, the membranous PKC- $\beta$ protein level was significantly elevated in BDL kidney; this was prevented by melatonin treatment (Figure 2a,d).

Next, we determined if melatonin-mediated increased uptake of L-arginine was regulated by PKC- $\alpha$ and PKC- $\beta$ differentially. As shown in Figure 3, melatonin treatment induced the uptake of L-arginine in clone 9 cells. Similarly, the uptake of L-arginine was induced by the inhibitor Gö 6976, which is specific to PKC- $\alpha$ and PKC- $\beta$, but not the PKC- $\beta$-specific inhibitor Ro-31-8220, indicating a role for PKC- $\alpha$ in melatonin-mediated increases of L-arginine uptake. In addition, cotreatment of melatonin and Gö 6976 increased L-arginine levels similarly to those treated with melatonin or Gö 6976 alone, suggesting that there was no synergistic effect. 
a

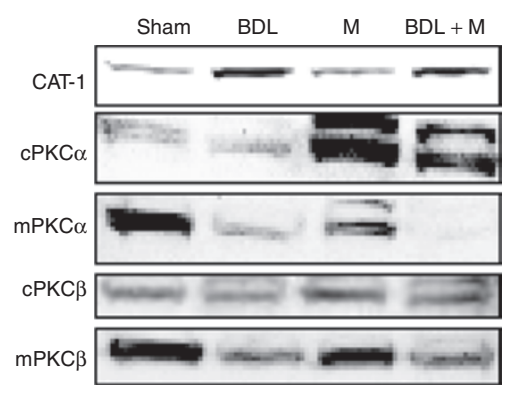

b

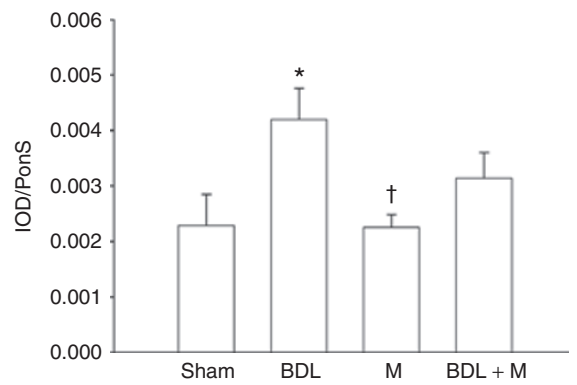

C
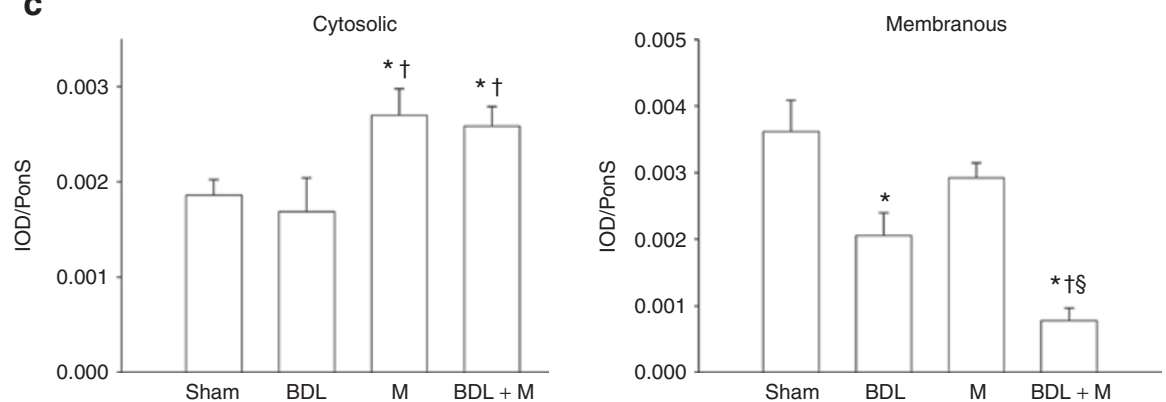

d

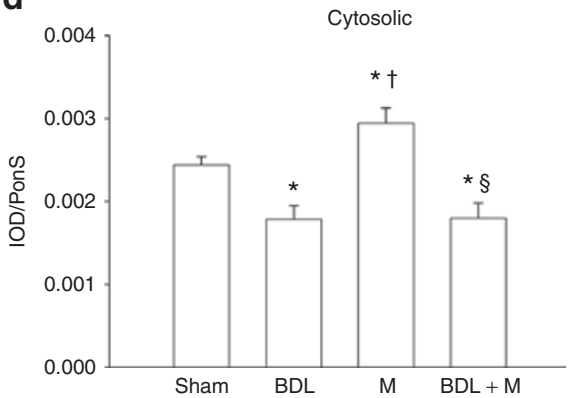

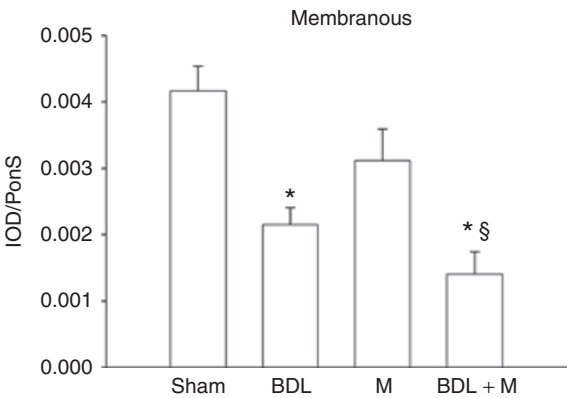

Figure 1. Protein levels of CAT-1, PKC- $\alpha$, and PKC- $\beta$ in the liver. (a) Representative western blots show (b) CAT-1 (68 kDa), cytosolic and membranous (c) PKC- $\alpha(82 \mathrm{kDa})$, and (d) PKC $-\beta(80 \mathrm{kDa})$ in the liver. Representative blots are shown in (a) and bar charts show quantitative results. ${ }^{*} P<0.05$ vs. sham; ${ }^{\dagger} P<$ 0.05 vs. $B D L ;{ }^{\ddagger} P<0.05$ vs. M. BDL, bile duct ligation; $B D L+M$, melatonin-treated $B D L$ rat; $C A T-1$, cationic amino acid transporter-1; IOD, integrated optical density; $M$, melatonin-treated sham; PKC, protein kinase $C$; PonS, Ponceau red staining.

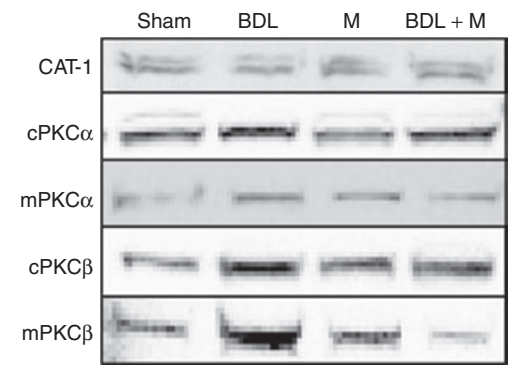

b

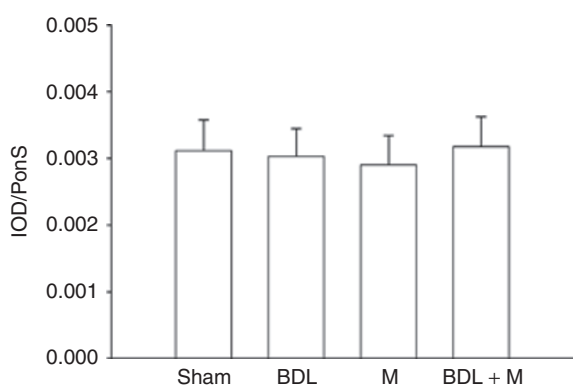

C
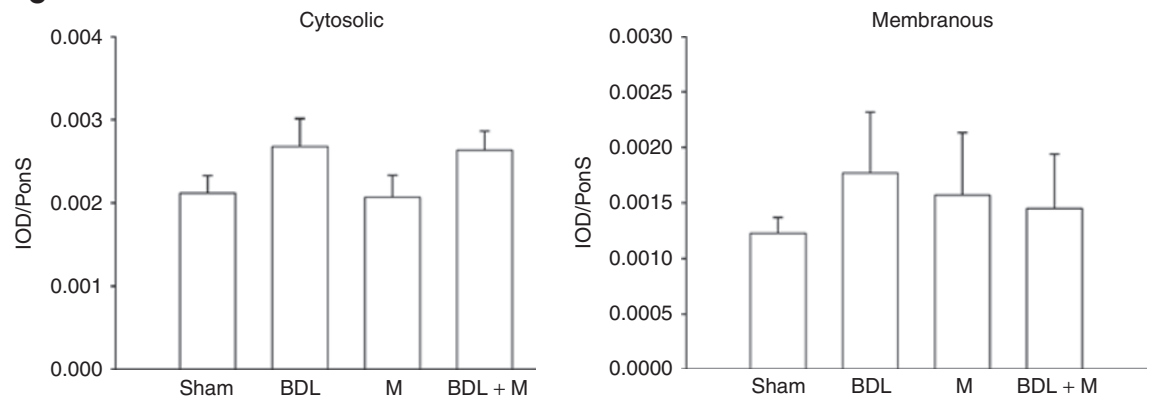

d

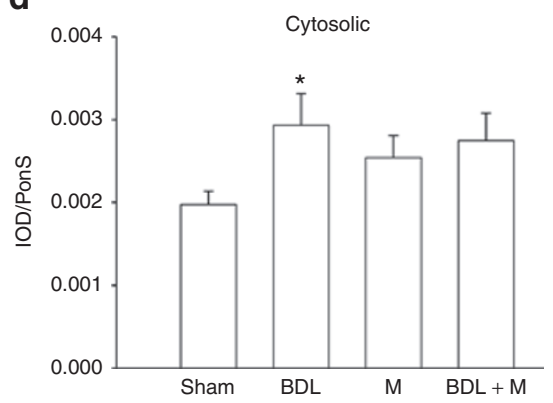

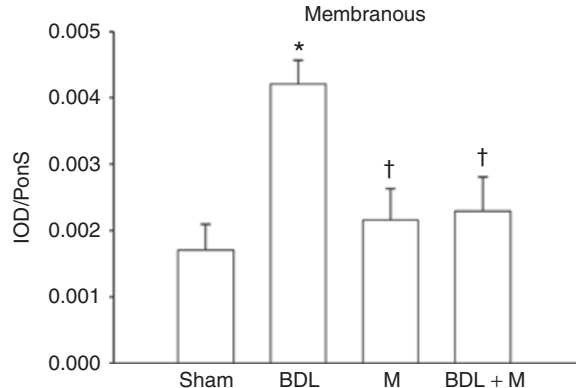

Figure 2. Protein levels of CAT-1, PKC- $\alpha$, and PKC- $\beta$ in the kidney. (a) Representative western blots show (b) CAT-1 (68 kDa), cytosolic and membranous (c) PKC- $\alpha(82 \mathrm{kDa})$, and (d) PKC- $\beta(80 \mathrm{kDa})$ in the kidney. Representative blots are shown in (a) and bar charts show quantitative results. ${ }^{*} P<0.05$ vs. sham; ${ }^{+} P<0.05$ vs. BDL. BDL, bile duct ligation; BDL $+M$, melatonin-treated BDL rat; CAT-1, cationic amino acid transporter-1; IOD, integrated optical density; $M$, melatonin-treated sham; PKC, protein kinase C; PonS, Ponceau red staining.

We further investigated whether melatonin increases $\mathrm{L}$-arginine availability because of the reduction of consumption via other metabolic pathways (e.g., arginase II) or increase of de novo synthesis (by ASS and ASL). Protein levels of arginase
II, ASS, and ASL were measured (Figure 4a). In the liver, the protein level of ASS was not different among the four groups (Figure 4b) whereas hepatic ASL protein level was higher in BDL group than in the sham group (Figure $4 c$ ). Renal arginase 
II level was lower in the $\mathrm{BDL}+\mathrm{M}$ group as compared with that in the sham and BDL groups (Figure 4d). In the kidney, protein levels of both ASS and ASL were not different among the four groups (Figure 4e,f).

Next, we found that cytosolic p47phox protein levels were not different among the four groups in both liver and kidney (Figure 5a). The p47phox protein levels in the membranous fraction were increased in BDL liver and kidney; this was

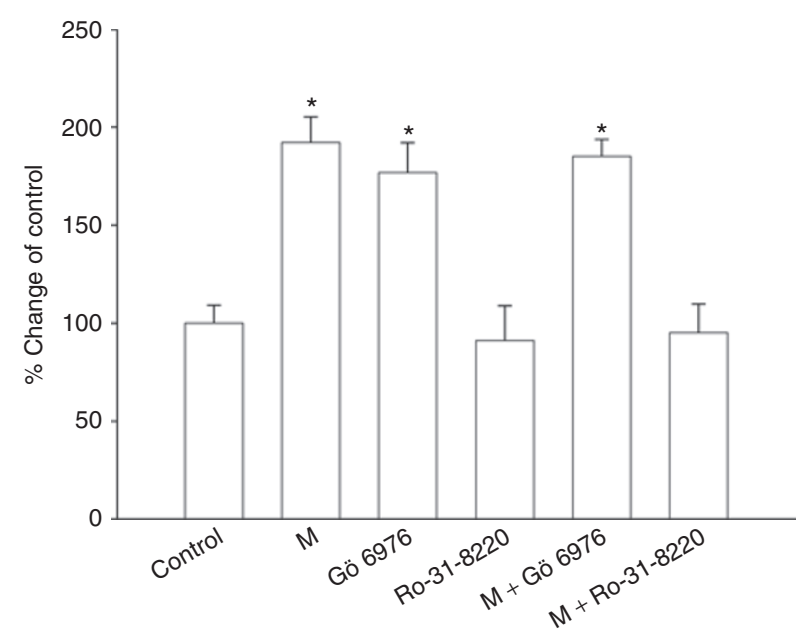

Figure 3. Effect of melatonin, inhibitor Gö 6976, which is specific to PKC- $\alpha$ and PKC- $\beta$, and PKC- $\beta$-specific inhibitor Ro-31-8220 on L-arginine uptake in clone 9 cells. Clone 9 cells were treated with melatonin $100 \mathrm{nmol} / \mathrm{l}$, in the absence or presence of Ro-31-8220, $1 \mu \mathrm{mol} / \mathrm{l}$, and Gö 6976, 1 $\mu \mathrm{mol} / \mathrm{l}$. All measurements were done in four runs. ${ }^{*} P<0.05$ vs. control. $M$, melatonin-treated sham; PKC, protein kinase $C$. prevented by melatonin therapy (Figure 5b,c). Similarly, renal NADPH-dependent superoxide production was significantly increased in BDL rats and was prevented by melatonin therapy (Figure 5d).

\section{DISCUSSION}

This study provides insight into a novel mechanism by which PKC- $\alpha$ and PKC- $\beta$ are differentially regulated by melatonin on CAT-mediated L-arginine transport and NADPH oxidaseinduced superoxide production in the liver and kidneys of young rats with BDL. Our major findings can be summarized as follows: (i) BDL-induced increases of $\mathrm{L}$-arginine in the liver relate to decreased PKC- $\alpha$ and PKC- $\beta$ translocation, increased CAT-1 protein levels, and increased ASL levels; (ii) melatonin increases L-arginine concentrations in BDL liver, which is correlated with decreased PKC- $\alpha$ translocation; (iii) in the BDL kidney, melatonin significantly decreases PKC- $\beta$ levels, reduces $\mathrm{p} 47$ phox translocation, and diminishes NADPHdependent superoxide production; (iv) melatonin increases $\mathrm{L}$-arginine concentrations in BDL kidneys, which is correlated with decreased renal level of arginase II but not PKC- $\alpha$ or CAT-1. Figure 6 is a simple scheme summarizing our results.

We have previously shown that melatonin lessens BDLinduced mortality and kidney injury in young rats through suppressing increased ADMA and oxidative stress $(2,19)$. L-Arginine competes with ADMA for NO synthase to generate NO, and L-arginine supplementation attenuates oxidative stress. In this study, we extended our previous finding and demonstrated that melatonin-mediated protective effects in rats with $\mathrm{BDL}$ are also related to the L-arginine pathway, a

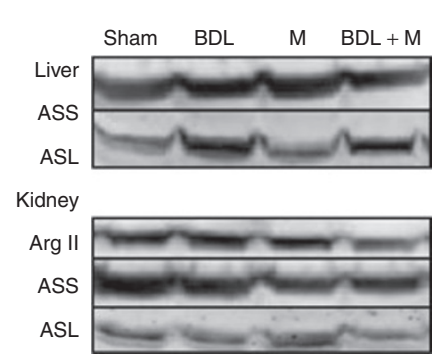

d

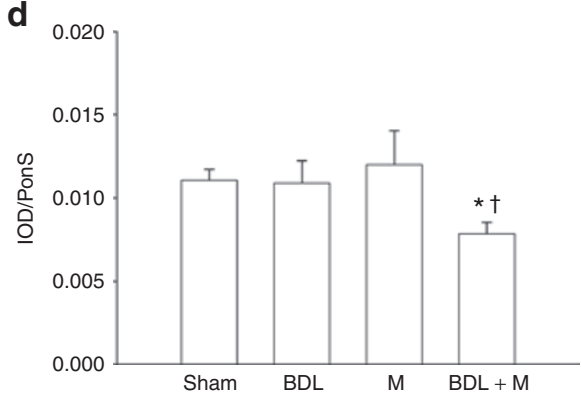

b

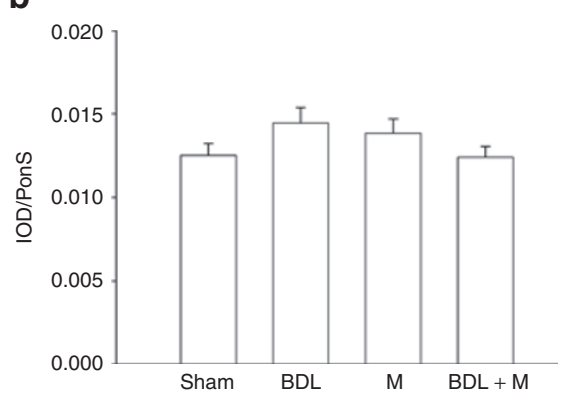

e

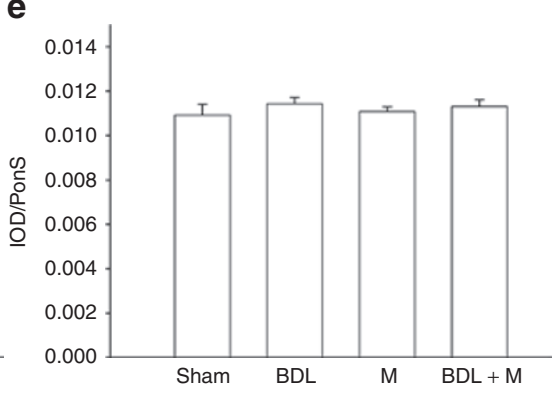

C

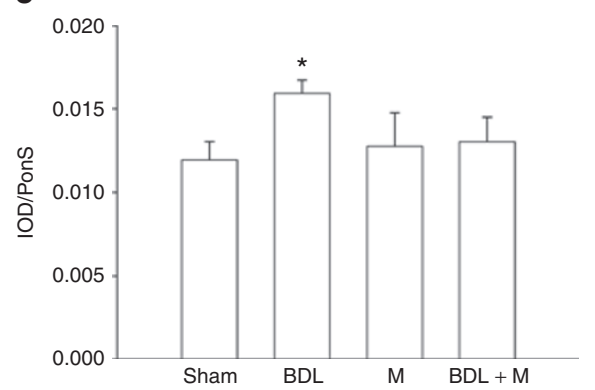

f

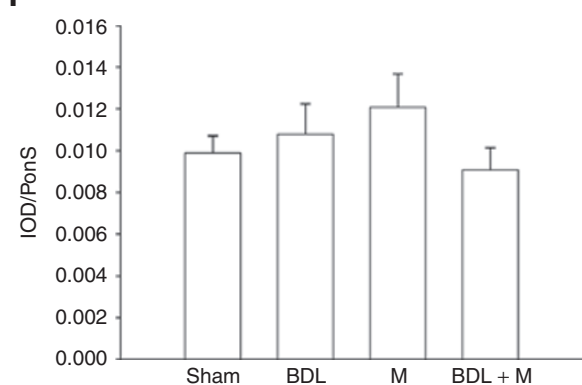

Figure 4. Effect of melatonin on protein levels of ASS, ASL, and arginase II. (a) Representative western blots show (b) ASS (50kDa) and (c) ASL (50 kDa) in the liver, and (d) arginase II (40 kDa), (e) ASS (50 kDa), and (f) ASL (50 kDa) in the kidney. Representative blots are shown in (a) and bar charts show quantitative results. ${ }^{*} P<0.05$ vs. sham; ${ }^{\dagger} P<0.05$ vs. BDL. Arg II, arginase Il; ASS, argininosuccinate synthase; ASL, argininosuccinate lyase; BDL, bile duct ligation; $\mathrm{BDL}+\mathrm{M}$, melatonin-treated BDL rat; CAT-1, cationic amino acid transporter-1; IOD, integrated optical density; $M$, melatonin-treated sham; PKC, protein kinase C; PonS, Ponceau red staining. 
a
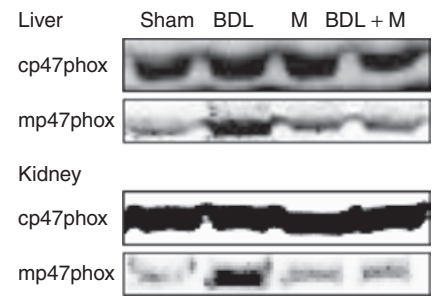

b

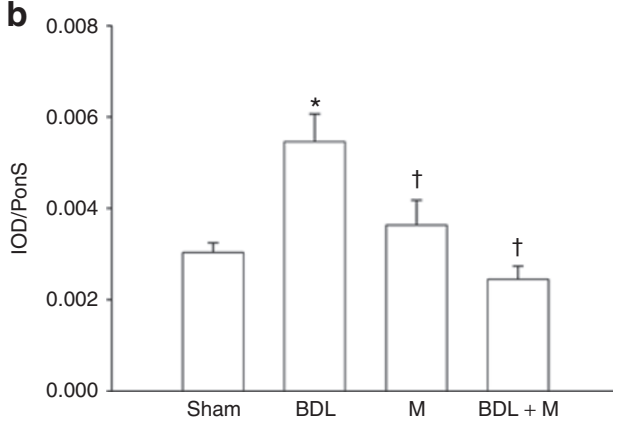

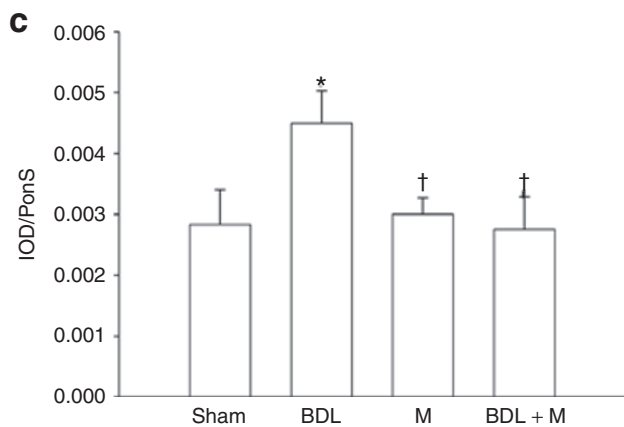

d

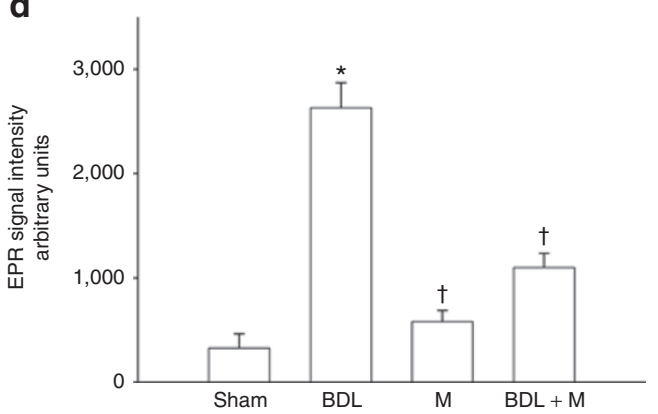

Figure 5. Effect of melatonin on protein levels of p47phox. (a) Representative western blots show cytosolic and membranous p47phox (47 kDa) in the (b) liver and (c) kidney; (d) NADPH-dependent superoxide production in the kidney detected by electron paramagnetic resonance (EPR). Representative blots are shown in (a) and bar charts show quantitative results. ${ }^{*} P<0.05$ vs. sham; ${ }^{+} P<0.05$ vs. BDL. BDL, bile duct ligation; BDL $+M$, melatonin-treated $\mathrm{BDL}$ rat; CAT-1, cationic amino acid transporter-1; IOD, integrated optical density; $\mathrm{M}$, melatonin-treated sham; NADPH, nicotinamide adenine dinucleotide phosphate; PKC, protein kinase C; PonS, Ponceau red staining.

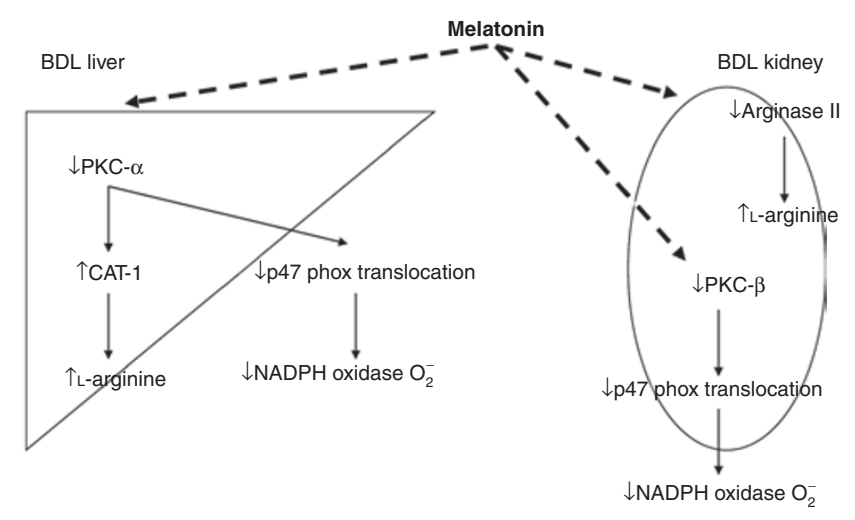

Figure 6. Simplified scheme showing the differential effects of melatonin on L-arginine and NADPH oxidase in the liver and kidney in rats with BDL. Dashed lines indicate the effects of melatonin on BDL rats: melatonin inhibits protein kinase C- $\alpha$ (PKC- $\alpha$ ) activity, consequently amplifying CAT1-mediated L-arginine uptake and decreasing NADPH oxidase in the BDL liver; melatonin inhibits PKC- $\beta$ activity to reduce NADPH oxidase-derived superoxide production in the BDL kidney; and melatonin reduces arginase II to increase L-arginine levels in the BDL kidney. BDL, bile duct ligation; CAT-1, cationic amino acid transporter-1; NADPH, nicotinamide adenine dinucleotide phosphate; PKC, protein kinase $\mathrm{C}$.

except for its beneficial effects on ADMA and oxidative stress. The central question of this study is how melatonin increases $\mathrm{L}$-arginine levels in BDL liver and kidneys.

We found that $\mathrm{L}$-arginine levels are elevated in the BDL liver. In this study, the reason for the increases of $\mathrm{L}$-arginine in rats with BDL is probably the increased uptake (i.e., increased CAT-1 expression) as well as increased de novo synthesis (i.e., increased
ASL expression) in the damaged liver. Of note, melatonin additionally increased hepatic L-arginine levels in young rats with $\mathrm{BDL}$. Inhibition of PKC- $\alpha$ has been reported to increase $\mathrm{L}$-arginine uptake (12). Our data demonstrated that PKC- $\alpha$ activation, as detected by translocation of cytosolic PKC- $\alpha$ activity to the membranous fraction, was inhibited by melatonin. We also found that melatonin and the inhibitor Gö 6976, which is specific to PKC- $\alpha$ and PKC- $\beta$, similarly increased intracellular $\mathrm{L}$-arginine levels in clone 9 cells. However, the PKC- $\beta$-specific inhibitor, Ro-31-8220, had no effect on melatonin-mediated $\mathrm{L}$-arginine uptake. These observations indicate that the melatonin inhibits PKC- $\alpha$ activity to increase CAT-1-mediated $\mathrm{L}$-arginine uptake. A previous study indicated that melatonin decreases PKC activity in glioma cells (17), which was supported by our data showing the possible role of melatonin as a PKC inhibitor. In addition, our data are in agreement with a previous report showing that PKC- $\alpha$ regulates CAT- 1 transport activity independent of its expression (13).

In line with our previous study (2), melatonin increases renal L-arginine concentrations and prevents kidney damage in young rats after $2 \mathrm{wk}$ of BDL. Unlike in the liver, the increase of $\mathrm{L}$-arginine is independent of the PKC- $\alpha / \mathrm{CAT}-1$ pathway in $\mathrm{BDL}$ kidneys. It seems possible that melatonin reduces consumption of L-arginine via arginase II to increase renal L-arginine levels. Because different cell types express different members of the PKC and CAT families for the delivery of L-arginine $(13,18)$, further study is needed to elucidate whether the protective role of melatonin on the L-arginine pathway might be due to its regulation of other PKC isoforms. 
We recently found that the levels of the subunit of NADPH oxidase, p22phox, were higher in BDL rats than in shams in both the liver and kidney (2). However, increased p22phox was not prevented by melatonin. Because activation of NADPH oxidase requires translocation of p47phox to bind p22phox, in this study, we further determined the effects of melatonin on p47phox translocation and NADPH oxidase-dependent superoxide production. It is noteworthy that melatonin significantly reduces BDL-induced PKC- $\beta$ translocation, decreases p47phox translocation, and diminishes NADPH-dependent superoxide production. Given that melatonin prevents BDL-induced kidney injury and oxidative stress (2), these findings support that the notion that melatonin may inhibit PKC- $\beta$ to suppress NADPH oxidase-induced ROS production, consequently protecting the kidney against BDL-induced damage. In addition, a previous report showing that PKC- $\alpha$ can also enhance superoxide production via NADPH oxidase assembly with the p47phox subunit (8), which was consistent with our study, demonstrated that melatonin reduced PKC- $\alpha$ and p47phox translocation concurrently in BDL liver. Therefore, these observations are in agreement with other studies showing that melatonin can reduce p47phox translocation to prevent NADPH oxidase assembly in a PKC-dependent manner $(20,21)$.

Melatonin has been demonstrated to be an efficient antioxidant with great clinical significance $(3,22)$. In addition to its ADMAlowering effect (2), our data demonstrate that the beneficial effects of melatonin include enhancement of tissue L-arginine levels and its action as a PKC inhibitor. Even though melatonin can increase L-arginine levels in BDL liver and kidney by different mechanisms, the plasma L-arginine levels are not different among the four groups. This finding suggests that plasma $\mathrm{L}$-arginine levels do not necessarily reflect tissue levels and that a therapeutic intervention in the $\mathrm{L}$-arginine/NO pathway must consider the complex regulation of interorgan L-arginine transport. Next, PKC inhibitors are expected to exert a wide range of pharmacological activities therapeutically beneficial for many diseases, including CLD $(23,24)$. Thus, our data further provide clinical evidence for the extended usefulness of melatonin in the future. A previous study demonstrated that melatonin reduced the increased serum total bile acid concentration, one of the indexes of cholestasis, in BDL-treated adult rats (25). Given that bile acids are implicated in the pathogenesis of cholestatic liver damage through oxidative stress (26), further studies are warranted to determine whether melatonin can reduce bile acids to regulate PKC-dependent oxidative stress.

In conclusion, differential mechanisms are involved in the melatonin-mediated increases of $\mathrm{L}$-arginine levels in the liver vs. the kidney: in the liver, melatonin inhibits PKC- $\alpha$ activity to increase CAT-1-mediated L-arginine uptake, whereas in the kidney, melatonin increases L-arginine level mainly by reducing consumption of L-arginine via arginase II. In addition, melatonin inhibits PKC- $\beta$ to reduce NADPH-dependent superoxide production, which may protect the BDL kidney against damage. Our findings highlight the potential therapeutic use of melatonin in children with CLD via regulation of $\mathrm{L}$-arginine and the PKC pathway.

\section{METHODS}

\section{Experimental Protocols}

This experiment was performed under the Guidelines for Animal Experiments of Chang Gung Memorial Hospital and Chang Gung University. Male and female Sprague Dawley rats were obtained (BioLASCO Taiwan, Taipei, Taiwan) for mating. Rats were housed and maintained in an Association for Assessment and Accreditation of Laboratory Animal Care International-accredited facility, with free access to tap water and standard rat chow. Male offspring rats at postnatal day $17 \pm 1$ weighing about $50 \mathrm{~g}$ were used. Obstructive jaundice was induced by opening a midline incision and ligating and dividing the common bile duct with double ligatures of the proximal duct as described previously (4).

Our previous study showed that the mortality rate of young rats with BDL was $\sim 30-40 \%(1,2)$, suggesting that we should a priori increase the number of animals in the BDL group to account for these losses. Thus, the rats receiving BDL were randomized into two groups: untreated ( $n=9, \mathrm{BDL}$ group) and melatonin-treated BDL rats $(n=6$, $\mathrm{BDL}+\mathrm{M}$ group). Melatonin-treated BDL rats received daily melatonin (Sigma, St. Louis, MO) $1 \mathrm{mg} / \mathrm{kg} / \mathrm{d}$ via i.p. injection in the light phase as described (2). The third group consisted of sham-operated rats receiving melatonin treatment $(n=6, \mathrm{M}$ group). In addition, another group of sham-operated rats were designated as the control group ( $n=6$, sham). All surviving rats were killed $14 \mathrm{~d}$ after surgery. Heparinized blood samples were collected, and the plasma was stored at $-80^{\circ} \mathrm{C}$ until assayed. The liver and kidneys were harvested after perfusion with phosphate-buffered saline. The functional parameters of liver and kidney, including aspartate transaminase, alanine transaminase, direct and total bilirubin, and creatinine levels, were measured as described previously (2).

\section{Detection of L-arginine and ADMA by High-Performance Liquid Chromatography}

L-Arginine and ADMA levels in plasma and tissue were measured using high-performance liquid chromatography (HP series 1100; Agilent Technologies, Santa Clara, CA) with the OPA-3MPA derivatization reagent as described previously (2). Briefly, $100 \mathrm{mg}$ of frozen liver or kidney tissue was homogenized in lysis buffer and centrifuged at $13,000 \mathrm{rpm}$ at $4^{\circ} \mathrm{C}$ for $30 \mathrm{~min}$. Tissue lysates were collected, and the protein concentration was measured using the Lowry method (DC Protein Assay, Bio-Rad, Hercules, CA). L-Homoarginine (Fluka, Neu Ulm, Germany) was used as the internal standard. Recovery was $\sim 90-105 \%$. The tissue concentration was factored in for protein concentration, which was represented as $\mu \mathrm{mol} / \mathrm{l} / \mathrm{mg}$ protein.

\section{Western Blot}

Western blot analysis was performed as described previously $(2,27)$. Briefly, measurement was conducted on the liver and kidneys (100$200 \mu \mathrm{g}$ total protein).

The protein abundances of PKC- $\alpha$, PKC- $\beta$, and p47phox were analyzed in both the membrane and cytoplasmic fractions to examine their translocation as described previously (27). For PKC, we used a mouse antirat PKC- $\alpha$ antibody (BD Transduction Laboratories, Lexington KY; 1:1,000 dilution, overnight incubation) and a mouse antirat PKC- $\beta$ antibody (BD Transduction Laboratories; 1:500 dilution, overnight incubation) followed by a secondary rabbit antimouse antibody. To detect the subunit of NADPH oxidase p47phox, we used a rabbit antirat p47phox antibody (Millipore, Billerica, MA; 1:500 dilution, overnight incubation). For ASS and ASL, a goat antirat ASS antibody (Santa Cruz Biotechnology, Santa Cruz, CA; 1:200 dilution, overnight incubation) and a rabbit antirat ASL antibody (Santa Cruz Biotechnology; 1:200 dilution, overnight incubation) were applied. For CAT-1, we used a rabbit antirat antibody (Abcam, Cambridge, MA; 1:250 dilution); for arginase II, we used a rabbit antibody (Santa Cruz Biotechnology; 1:1,000 dilution, overnight incubation) followed by a goat antirabbit secondary antibody. Bands of interest were visualized using ECL reagents (PerkinElmer, Waltham, MA) and quantified by densitometry (Quantity One Analysis software; Bio-Rad) as integrated optical density after subtraction of background. The integrated optical density was factored for Ponceau red staining (PonS) to correct for any variations in 
total protein loading. The protein abundance was represented as integrated optical density/PonS.

\section{Detection of PKC-Dependent L-arginine Uptake}

A normal rat hepatocyte cell line (clone 9) derived from normal Sprague Dawley rat liver tissue was purchased from Bioresource Collection and Research Center (BCRC No. 60201, Hsin-Chu, Taiwan). Cells were maintained in F-12 K medium (Sigma Chemical, St. Louis, MO) supplemented with heat-inactivated fetal calf serum (Hyclone, Logan, UT), penicillin, and streptomycin (Invitrogen) as previously described (20). Twenty-four hours before experiments, cells were trypsinized and plated on $3.5-\mathrm{cm}$ diameter dishes or 96-well microplates. Clone 9 cells were treated with vehicle or melatonin $(100 \mathrm{nmol} / \mathrm{l})$ for $60 \mathrm{~min}$, in the absence or presence of the PKC- $\beta$ inhibitor (Ro-31-8220, $1 \mu \mathrm{mol} / \mathrm{l}$, Calbiochem, Merck, Darmstadt, Germany) and the $\mathrm{Ca}^{2+}$-dependent

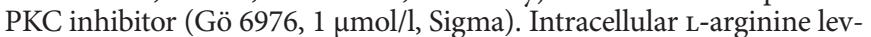
els were determined by high-performance liquid chromatography. The amount of L-arginine uptake was factored by control and represented as percentage change of control to reflect CAT activity.

\section{NADPH-Dependent Superoxide Production by Electron Paramagnetic Resonance}

NADPH-dependent superoxide production was measured by electron paramagnetic resonance spectroscopy with hydroxylamine spin probe 1-hydroxy-3-carboxypyrrolidine, as we previously described (27). Membrane sample from kidney was prepared, and $10 \mu \mathrm{g}$ of protein was added to $1 \mathrm{mmol} / 1$-hydroxy-3-carboxypyrrolidine, $200 \mu \mathrm{mol} / \mathrm{l}$ $\mathrm{NADPH}$, and $0.1 \mathrm{mmol} / \mathrm{l}$ diethylenetriaminepentaacetic acid in a total volume of $100 \mu \mathrm{l}$ of Chelex-treated phosphate-buffered saline. In duplicate samples, NADPH was omitted. Samples were placed in a $50 \mu$ l glass capillary tubes (Wilmad Glass, Buena, NJ). The electron paramagnetic resonance spectra were recorded using an EMX Plus EPR spectrometer (Bruker Biospin, Rheinstetten, Germany) equipped with a EMX-m40X microwave bridge operating at $9.87 \mathrm{GHz}$.

\section{Statistical Analysis}

Morphological parameters and biochemical parameters were analyzed by one-way ANOVA with a post hoc Fisher's least significant difference test. All analyses were performed using Statistical Package for the Social Sciences software (SPSS, Chicago, IL). Values were expressed as mean \pm SE. Significance was defined as $P<0.05$ for all tests.

\section{ACKNOWLEDGMENTS}

We thank Samuel H.H. Chan and the Center for Translational Research in Biomedical Sciences, Kaohsiung Chang Gung Memorial Hospital, for providing space to support electron paramagnetic resonance.

\section{STATEMENT OF FINANCIAL SUPPORT}

This work was supported by grant NHRI-EX101-9826SC from the National Health Research Institutes, Taiwan, and grant CMRPG890202 from the Kaohsiung Chang Gung Memorial Hospital, Taiwan.

\section{REFERENCES}

1. Huang LT, Tiao MM, Tain YL, Chen CC, Hsieh CS. Melatonin ameliorates bile duct ligation-induced systemic oxidative stress and spatial memory deficits in developing rats. Pediatr Res 2009;65:176-80.

2. Tain YL, Hsieh CS, Chen CC, Sheen JM, Lee CT, Huang LT. Melatonin prevents increased asymmetric dimethylarginine in young rats with bile duct ligation. J Pineal Res 2010;48:212-21.

3. Tan DX, Manchester LC, Terron MP, Flores LJ, Reiter RJ. One molecule, many derivatives: a never-ending interaction of melatonin with reactive oxygen and nitrogen species? J Pineal Res 2007;42:28-42.

4. Genade S, Genis A, Ytrehus K, Huisamen B, Lochner A. Melatonin receptor-mediated protection against myocardial ischaemia/reperfusion injury: role of its anti-adrenergic actions. J Pineal Res 2008;45:449-58.

5. Wu G, Morris SM Jr. Arginine metabolism: nitric oxide and beyond. Biochem J 1998;336 (Pt 1):1-17.
6. Pandi-Perumal SR, Srinivasan V, Maestroni GJ, Cardinali DP, Poeggeler B, Hardeland R. Melatonin: nature's most versatile biological signal? FEBS J 2006;273:2813-38.

7. Sampson SR, Lupowitz Z, Braiman L, Zisapel N. Role of protein kinase Calpha in melatonin signal transduction. Mol Cell Endocrinol 2006;252:82-7.

8. Herrera M, Silva GB, Garvin JL. Angiotensin II stimulates thick ascending limb superoxide production via protein kinase C(a)-dependent NADPH oxidase activation. J Biol Chem 2010;285:21323-8.

9. Nitti M, Pronzato MA, Marinari UM, Domenicotti C. PKC signaling in oxidative hepatic damage. Mol Aspects Med 2008;29:36-42.

10. Pan M, Stevens BR. Protein kinase C-dependent regulation of $\mathrm{L}$-arginine transport activity in Caco-2 intestinal cells. Biochim Biophys Acta 1995;1239:27-32.

11. Rotmann A, Strand D, Martiné U, Closs EI. Protein kinase C activation promotes the internalization of the human cationic amino acid transporter hCAT-1. A new regulatory mechanism for hCAT-1 activity. J Biol Chem 2004;279:54185-92.

12. Zharikov SI, Krotova KY, Belayev L, Block ER. Pertussis toxin activates $\mathrm{L}$-arginine uptake in pulmonary endothelial cells through downregulation of PKC-alpha activity. Am J Physiol Lung Cell Mol Physiol 2004;286:L974-83.

13. Krotova KY, Zharikov SI, Block ER. Classical isoforms of PKC as regulators of CAT-1 transporter activity in pulmonary artery endothelial cells. Am J Physiol Lung Cell Mol Physiol 2003;284:L1037-44.

14. Kitada M, Koya D, Sugimoto T, et al. Translocation of glomerular p47phox and p67phox by protein kinase C-beta activation is required for oxidative stress in diabetic nephropathy. Diabetes 2003;52:2603-14.

15. Meier M, Menne J, Haller $\mathrm{H}$. Targeting the protein kinase $\mathrm{C}$ family in the diabetic kidney: lessons from analysis of mutant mice. Diabetologia 2009;52:765-75.

16. Rimler A, Jockers R, Lupowitz Z, Sampson SR, Zisapel N. Differential effects of melatonin and its downstream effector PKCalpha on subcellular localization of RGS proteins. J Pineal Res 2006;40:144-52.

17. Martín V, Herrera F, García-Santos G, et al. Involvement of protein kinase $C$ in melatonin's oncostatic effect in C6 glioma cells. J Pineal Res 2007;43:239-44.

18. Li J, Gobe G. Protein kinase $\mathrm{C}$ activation and its role in kidney disease. Nephrology (Carlton) 2006;11:428-34.

19. Tain YL, Kao YH, Hsieh CS, et al. Melatonin blocks oxidative stressinduced increased asymmetric dimethylarginine. Free Radic Biol Med 2010;49:1088-98.

20. Zhou J, Zhang S, Zhao X, Wei T. Melatonin impairs NADPH oxidase assembly and decreases superoxide anion production in microglia exposed to amyloid-beta1-42. J Pineal Res 2008;45:157-65.

21. Ungvari Z, Csiszar A, Huang A, Kaminski PM, Wolin MS, Koller A. High pressure induces superoxide production in isolated arteries via protein kinase C-dependent activation of NAD(P)H oxidase. Circulation 2003;108:1253-8.

22. Tengattini S, Reiter RJ, Tan DX, Terron MP, Rodella LF, Rezzani R. Cardiovascular diseases: protective effects of melatonin. J Pineal Res 2008;44:16-25.

23. Rask-Madsen C, King GL. Proatherosclerotic mechanisms involving protein kinase $\mathrm{C}$ in diabetes and insulin resistance. Arterioscler Thromb Vasc Biol 2005;25:487-96.

24. Yang JI, Yoon JH, Bang YJ, et al. Synergistic antifibrotic efficacy of statin and protein kinase $\mathrm{C}$ inhibitor in hepatic fibrosis. Am J Physiol Gastrointest Liver Physiol 2010;298:G126-32.

25. Ohta Y, Kongo M, Kishikawa T. Melatonin exerts a therapeutic effect on cholestatic liver injury in rats with bile duct ligation. J Pineal Res 2003;34:119-26.

26. Muchova L, Vanova K, Zelenka J, et al. Bile acids decrease intracellular bilirubin levels in the cholestatic liver: implications for bile acid-mediated oxidative stress. J Cell Mol Med 2011;15:1156-65.

27. Tain YL, Hsu CN, Huang LT, Lau YT. Apocynin attenuates oxidative stress and hypertension in young spontaneously hypertensive rats independent of ADMA/NO pathway. Free Radic Res 2012;46:68-76. 